\title{
Thermochemistry of Heteroatomic Compounds: analysis and calculation of thermodynamic functions of organometallic compounds of I-IV groups of Mendeleev's Periodic table
}

\author{
Vitaly Vitalevich Ovchinnikov \\ Department of General Chemistry and Ecology, Tupolev Kazan National Researching Technical University, Tatarstan, Russia
}

Email address:

chem_vvo@mail.ru

\section{To cite this article:}

Vitaly Vitalevich Ovchinnikov. Thermochemistry of Heteroatomic Compounds: Analysis and Calculation of Thermodynamic Functions of Organometallic Compounds of I-IV Groups of Mendeleev's Periodic Table, American Journal of Physical Chemistry,

Vol. 2, No. 3, 2013, pp. 52-59. doi: 10.11648/j.ajpc.20130203.11

\begin{abstract}
It is necessary to note, that the heat of vaporization, all thermodynamic functions $\Delta_{\mathrm{c}, \mathrm{f}} G^{\mathrm{o}}, \Delta_{\mathrm{c}, \mathrm{f}} H^{\mathrm{o}}, S_{\mathrm{o}}, \Delta_{\mathrm{c}} S_{\text {cond }}^{\mathrm{o}}$ and heat capacity $\left(C_{\mathrm{p}}\right)$ of organometallic compounds of I-IV groups of Mendeleev's Periodic Table can be well characterized with the number of valence electrons $N$ of them. It is difficult to do some conclusions relatively stoichiometric coefficients $i$ and $f$ in modified by us Kharasch equation $\Delta_{\mathrm{vap}, \mathrm{c}, \mathrm{f}, \mathrm{s}} \Psi^{\circ}=i \pm f^{*} N$ as they concern to organometallic compounds of the different groups. Such conclusion can be made on settlement data of I and II groups only in our opinion. Nevertheless, 43 equations of this type have been created for the processes of vaporization, combustion, formation, entropic transformations and heat capacity. Probably, that such data will help with forecasting of thermochemical functions and parameters of yet not investigated organometallic compounds.
\end{abstract}

Keywords: Organometallic Compounds, Free Energy of Combustion, Free Energy of Formation, Entropy, Heat Capacity, Heat of Combustion Heat of Formation

\section{Introduction}

We have shown earlier [1,2], that the changes of thermodynamic functions $\Psi^{\circ}\left(\Delta \mathrm{G}^{\mathrm{o}}, \Delta H^{\mathrm{o}}, \Delta S^{\mathrm{o}}, C p\right)$, describing processes of vaporization (vap), combustion (c), formation (f), transfer of the heat from a hot body to cold body (entropy, s) and heat capacity various organic and biochemical compounds ( $\mathrm{Cp}$ ), can be effectively calculated on the equation (1), which is similarly to known Kharasch equation [3]. In equation (1) $i$ and $f$ are stoichiometric coefficients, $N$ is a number of valence electrons in the researching molecules, from which the number $h$ of lone electron pairs $(g=2)$ of heteroatoms is substracted or increased $[4,5]$

$$
\Delta_{\text {vap,c,f,s }} \Psi^{o}=i+f^{*}(N \pm h g)
$$

Necessary to add, that in accordance to Kharasch conception "The electronic conception of valence and heats of combustion of organic compounds" [3] the lone electron pairs should not take part in combustion process, apparently. We have chosen the $\mathrm{IV}^{\text {th }}$ group of Periodic Table (compounds of carbon and below) as a base point since in these compounds are not present a lone electron pairs $(h g=$ $0)$. According to this position the parameter $h$ is equal -1 and $h g=-2$, для compounds of $\mathrm{V}^{\text {th }}$ group the parameter $h$ is equal -2 and $h g=-4$ for $\mathrm{VI}^{\text {th }}$ group and the parameter $h$ is equal -3 and $h g=-6$ for $\mathrm{VII}^{\text {th }}$ group of Periodic system. Previously we informed [4], that for organic compounds of IIII groups, having a vacant s- and p-orbitals, necessary to add one, two and three lone electron pairs [4].

But after the careful analysis of valence conditions of metallic atoms of first three groups of Periodic system, we believe that it is not necessary to change of valence of metal (Me) in organometallic compounds of I-IV groups and it is not necessary to add of lone electron pairs on vacant s-and porbitals. Therefore the equation (2) represents a new, modified form of the equation (1) which has been used by us for calculations of thermodynamic functions organometall compounds of I-IV groups of Mendeleev's Periodic Table.

$$
\Delta_{\text {vap, }, \mathrm{f}, \mathrm{s}, \mathrm{s}} \Psi^{\mathrm{o}}=i \pm f^{*} N
$$




\section{Results and Discussion}

\subsection{Organic Compounds of I-II Groups (Lithium, zinc, Cadmium, Mercury)}

The processes of combustion of all these types of organometallic compounds in condensed phase of I-II groups (I-XXII, Table 1) may be presented by the general equation (3) [5]

$$
\mathrm{C}_{\mathrm{a}} \mathrm{H}_{\mathrm{b}} \mathrm{Me}_{\mathrm{c}} \text { (cond) }+(4 \mathrm{a}+\mathrm{b}) / 2 \mathrm{O}_{2} \rightarrow \mathrm{a} \mathrm{CO} 2 \text { (gas) }+\mathrm{b} / 2 \mathrm{H}_{2} \mathrm{O}+\mathrm{Me}_{\mathrm{c}(+1)} \mathrm{O} \text { (sol) }
$$

here $\mathrm{Me}$ are $\mathrm{Li}, \mathrm{Zn}, \mathrm{Cd}$ and $\mathrm{Hg} ; \mathrm{a}, \mathrm{b}, \mathrm{c}$ are stoichiometric coefficients. Necessary thermodynamic data for metal oxides are taken from monograph [28].

Necessary to note that the heat of combustion is more measured and studied than other thermodynamic functions and heat capacity [5].

\subsubsection{Lithium Compounds}

For the some lithium-organic of compounds (I-VI) are known the heats of vaporization, combustion and formation in condensed and gaseous phases only [6-9]. However these functions can be characterized only by two Eqs. (4 and 5), they are presented in the Table 2 also)

$$
\begin{aligned}
& \Delta_{\mathrm{c}} H^{\mathrm{o}}=(-249.6 \pm 28.8)-(106.9 \pm 1.4) N r 0.999, S_{\mathrm{o}} 11.9, n 3 \text { (compounds I-III) } \\
& \Delta_{\mathrm{f}} H^{\mathrm{o}} \text { cond }=(-1053.1 \pm 87.9)+(36.8 \pm 3.2) N r 0.999, S_{\mathrm{o}} 11.3, n 4 \text { (compounds III-VI) }
\end{aligned}
$$

\subsubsection{Zinc Compounds}

For Zn-organic compounds it is possible to present the Eqs. (6-9) from which the first characterizes the heats of vaporization. For (VII and VIII) compounds of this group the values of entropy and the heat of capacity in condensed state (Table 1) are known. This circumstance enables to calculate the values of combustion entropy $\left(\Delta_{\mathrm{c}} S^{0}\right)$ on the Hess law (Eq. 10) and then calculate the free energy of combustion and formation $\left(\Delta_{\mathrm{c}, \mathrm{f}} G^{0}\right)$ on the Gibbs's Eqs. (11 and 12). All calculated values are summed in Table 1.

$$
\begin{aligned}
& \Delta_{\text {vap }} H^{\mathrm{o}}=(19.7 \pm 2.1)+(0.7 \pm 0.05) N r 0.993, S_{\mathrm{o}} 1.5, n 4 \text { (compounds VII-X) } \\
& \Delta_{\mathrm{c}} H^{\mathrm{o}}=(-286.5 \pm 29.5)-(109.3 \pm 0.8) N r 0.999, S_{\mathrm{o}} 21.6, n 4 \text { (compounds VII-X) } \\
& \Delta_{\mathrm{f}} H_{\text {cond }}^{\mathrm{o}}=(102.7 \pm 28.9)-(3.9 \pm 0.8) N r 0.961, S_{\mathrm{o}} 21.2, n 4 \text { (compounds VII-X) } \\
& \Delta_{\mathrm{f}} H^{\mathrm{o}} \text { gas }=(122.1 \pm 31.8)-(3.3 \pm 0.9) N r 0.937, S_{\mathrm{o}} 23.4, n 4 \text { (compounds VII-X) }
\end{aligned}
$$

\begin{tabular}{|c|c|c|c|c|c|c|c|c|c|c|}
\hline \multirow[b]{2}{*}{$\begin{array}{l}\text { No } \\
\text { comp. }\end{array}$} & \multirow[b]{2}{*}{$\begin{array}{l}\text { Title of Compound, formula, } \\
N\end{array}$} & \multicolumn{2}{|c|}{ Free energy } & \multicolumn{4}{|c|}{ Enthalpy (heat contributions) } & \multicolumn{3}{|c|}{ Entropy and heat capacity } \\
\hline & & $\begin{array}{l}-\Delta_{\mathrm{c}} G^{\mathrm{o}} \\
(\text { Calc. } \\
\pm 0.5)^{\mathrm{a}}\end{array}$ & $\begin{array}{l}-\Delta_{\mathrm{f}} G^{\mathrm{o}} \\
(\text { Calc. } \\
\pm 0.5)^{\mathrm{b}}\end{array}$ & $-\Delta_{\text {vap }} H^{\circ}$ & $-\Delta_{\mathrm{c}} H^{\mathrm{o}}$ & $-\Delta_{\mathrm{f}} H^{\circ}$ & $-\Delta_{\mathrm{f}} H_{\text {gas }}^{\mathrm{o}}$ & $S^{\circ}$ & $\begin{array}{l}-\Delta_{\mathrm{c}} S^{\mathrm{o}} \\
(\text { Calc. } \\
\pm 0.5)^{\mathrm{f}}\end{array}$ & $C_{p}$ \\
\hline 1 & 2 & 3 & 4 & 5 & 6 & 7 & 8 & 9 & 10 & 11 \\
\hline \multicolumn{11}{|c|}{ Lithium compounds } \\
\hline I & Ethyllithium, $\mathrm{C}_{2} \mathrm{H}_{5} \mathrm{Li}, 14$ & & & $\begin{array}{l}116.6^{[6]} \\
\pm 0.8\end{array}$ & $\begin{array}{l}1741.8 \\
{[6]} \\
\pm 5.4\end{array}$ & & $\begin{array}{l}-63.7^{[8]} \\
\pm 2.2\end{array}$ & & & \\
\hline II & $\begin{array}{l}\text { 2-Methylethyllithium, } \\
\mathrm{C}_{3} \mathrm{H}_{7} \mathrm{Li}, 20\end{array}$ & & & & $\begin{array}{l}2398.0 \\
{[7]}\end{array}$ & & & & & \\
\hline III & Buthyllithium, $\mathrm{C}_{4} \mathrm{H}_{9} \mathrm{Li}, 26$ & & & $\begin{array}{l}107.1^{[6]} \\
\pm 2.9\end{array}$ & $\begin{array}{l}3025.0 \\
{[6] \pm 7.1}\end{array}$ & $\begin{array}{l}109.5 \\
{\left[{ }^{8}\right] \pm 3.2}\end{array}$ & $\begin{array}{l}2.4^{[8]} \\
\pm 4.3\end{array}$ & & & \\
\hline IV & $\begin{array}{l}\text { 2-Methylpropyllithium, } \mathrm{C}_{4} \mathrm{H}_{9} \mathrm{Li} \text {, } \\
26\end{array}$ & & & & & $\begin{array}{l}88.2^{[7]} \\
\pm 3.2^{2}\end{array}$ & & & & \\
\hline V & $\begin{array}{l}\text { 2,2-Dimethylethyllithium, } \\
\mathrm{C}_{4} \mathrm{H}_{9} \mathrm{Li}, 26\end{array}$ & & & & & $\begin{array}{l}92.5^{[7]} \\
\pm 2.4 \\
-50.4\end{array}$ & & & & \\
\hline VI & Phenyllithium, $\mathrm{C}_{6} \mathrm{H}_{5} \mathrm{Li}, 30$ & & & & & $\begin{array}{l}{[7]} \\
\pm 2.1\end{array}$ & & & & \\
\hline \multicolumn{11}{|c|}{ Zinc compounds } \\
\hline VII & Dimethylzinc, $\mathrm{C}_{2} \mathrm{H}_{6} \mathrm{Zn}, 16$ & $\begin{array}{l}1918.4 \\
\pm 9.6\end{array}$ & $\begin{array}{l}85.1 \\
\pm 0.4\end{array}$ & $\begin{array}{l}29.5^{[5]} \\
\pm 0.4\end{array}$ & $\begin{array}{l}2020.0 \\
{[5] \pm 5.9}\end{array}$ & $\begin{array}{l}-25.0 \\
{[8]} \\
\pm 5.9\end{array}$ & $\begin{array}{l}-52.9^{[8]} \\
\pm 1.3\end{array}$ & $201.6^{[9]}$ & $\begin{array}{l}340.6 \\
\pm 1.7\end{array}$ & $\begin{array}{l}129.2 \\
{[9]}\end{array}$ \\
\hline VIII & Diethylzinc, $\mathrm{C}_{4} \mathrm{H}_{10} \mathrm{Zn}, 28$ & $\begin{array}{l}3251.6 \\
\pm 16.2\end{array}$ & $\begin{array}{l}104.5 \\
\pm 0.5\end{array}$ & $\begin{array}{l}40.2^{[5]} \\
\pm 2.1\end{array}$ & $\begin{array}{l}3372.3 \\
{[5] \quad \pm 2.1}\end{array}$ & $\begin{array}{l}-18.0^{\mathrm{c}} \\
\pm 6.0^{\mathrm{C}}\end{array}$ & $\begin{array}{l}-57.0^{c} \\
\pm 6.0\end{array}$ & $\begin{array}{l}290.2 \\
{[10]}\end{array}$ & $\begin{array}{l}404.7 \\
\pm 2.0\end{array}$ & $\begin{array}{l}194.4 \\
{[10]}\end{array}$ \\
\hline IX & Dipropylzinc, $\mathrm{C}_{6} \mathrm{H}_{14} \mathrm{Zn}, 40$ & & & $\begin{array}{l}45.6^{[5]} \\
\pm 2.5\end{array}$ & $\begin{array}{l}4653.0 \\
{[5]} \\
\pm 23.0\end{array}$ & $\begin{array}{l}59.0^{[8]} \\
\pm 23.0\end{array}$ & $\begin{array}{l}17.0^{[8]} \\
\pm 23.0\end{array}$ & & & \\
\hline $\mathrm{X}$ & Dibutylzinc, $\mathrm{C}_{8} \mathrm{H}_{18} \mathrm{Zn}, 52$ & & & $54.4^{[5]}$ & 5965.0 & 106.0 & $55.0^{[8]}$ & & & \\
\hline
\end{tabular}

Table 1. Thermodynamic functions $\left(\mathrm{kJ} \mathrm{mol}^{-1}, \mathrm{~J} \mathrm{~mol}^{-1} \mathrm{~K}^{-1}\right)$ of organometallic compounds at $\mathrm{P} 101 \mathrm{kPa}$ and $298.15 \mathrm{~K}$; all compounds are in condensed state 


\begin{tabular}{|c|c|c|c|c|c|c|c|c|c|c|}
\hline \multirow[b]{2}{*}{$\begin{array}{l}\text { No } \\
\text { comp. }\end{array}$} & \multirow[b]{2}{*}{$\begin{array}{l}\text { Title of Compound, formula, } \\
N\end{array}$} & \multicolumn{2}{|c|}{ Free energy } & \multicolumn{4}{|c|}{ Enthalpy (heat contributions) } & \multicolumn{3}{|c|}{ Entropy and heat capacity } \\
\hline & & $\begin{array}{l}-\Delta_{\mathrm{c}} G^{0} \\
(\text { Calc. } \\
\pm 0.5)^{\text {a }} \\
\end{array}$ & $\begin{array}{l}-\Delta_{\mathrm{f}} G^{\mathrm{o}} \\
(\text { Calc. } \\
\pm 0.5)^{\mathrm{b}} \\
\end{array}$ & $-\Delta_{\text {vap }} H^{\circ}$ & $-\Delta_{\mathrm{c}} H^{\mathrm{o}}$ & $-\Delta_{\mathrm{f}} H^{\mathrm{o}}$ & $-\Delta_{\mathrm{f}} H_{\mathrm{gas}}^{\mathrm{o}}$ & $S^{\mathrm{o}}$ & $\begin{array}{l}-\Delta_{S} S^{\circ} \\
(\text { Calc. } \\
\pm 0.5)^{\mathrm{f}} \\
\end{array}$ & $C_{p}$ \\
\hline 1 & 2 & 3 & 4 & $\begin{array}{l}5 \\
\pm 3.3\end{array}$ & $\begin{array}{l}6 \\
{[5]} \\
\pm 23.0\end{array}$ & $\begin{array}{l}7 \\
{[8]} \\
\pm 23.0\end{array}$ & $\begin{array}{l}8 \\
\pm 23.0\end{array}$ & 9 & 10 & 11 \\
\hline \multicolumn{11}{|c|}{ Cadmium compounds } \\
\hline XI & Dimethylcadmium, $\mathrm{C}_{2} \mathrm{H}_{6} \mathrm{Cd}, 16$ & $\begin{array}{l}1890.7 \\
\pm 9.4\end{array}$ & $\begin{array}{l}127.9 \\
\pm 1.6\end{array}$ & $\begin{array}{l}37.1^{\mathrm{d}} \\
\pm 0.1^{-}\end{array}$ & $\begin{array}{l}1988.0 \\
{[5]} \\
\pm 12.0\end{array}$ & $\begin{array}{l}67.7^{[8]} \\
\pm 1.3\end{array}$ & $\begin{array}{l}104.8^{[8]} \\
\pm 1.3\end{array}$ & $201.88^{\mathrm{e}}$ & $\begin{array}{l}329.6 \\
\pm 1.6\end{array}$ & ${ }_{\mathrm{e}}^{132.01}$ \\
\hline XII & Diethylcadmium, $\mathrm{C}_{4} \mathrm{H}_{10} \mathrm{Cd}, 28$ & & & $\begin{array}{l}46.0^{[5]} \\
\pm 1.9^{[27]} \\
54.2^{[27}\end{array}$ & $\begin{array}{l}3349.7 \\
\quad \pm 3.3\end{array}$ & $\begin{array}{l}59.3^{[8]} \\
\pm 1.9\end{array}$ & $\begin{array}{l}105.3^{[8]} \\
\pm 1.9\end{array}$ & & & \\
\hline XIII & Dipropylcadmium, $\mathrm{C}_{6} \mathrm{H}_{14} \mathrm{Cd}, 40$ & & & \pm 0.4 & $\begin{array}{l}4710.8 \\
{ }^{[4]} \pm 23.5\end{array}$ & & & & & \\
\hline XIV & Dibutylcadmium, $\mathrm{C}_{8} \mathrm{H}_{18} \mathrm{Cd}, 52$ & & & $\begin{array}{l}67.7^{[27]} \\
\pm 1.2\end{array}$ & & & & & & \\
\hline \multicolumn{11}{|c|}{ Mercury compounds } \\
\hline $\mathrm{XV}$ & Dimethylmercury, $\mathrm{C}_{2} \mathrm{H}_{6} \mathrm{Hg}, 16$ & & & $\begin{array}{l}34.6^{[5]} \\
\pm 0.8\end{array}$ & $\begin{array}{l}1806.7 \\
{[5]}\end{array}$ & $\begin{array}{l}-57.8 \\
{[8]} \\
\pm 3.0\end{array}$ & $\begin{array}{l}-92.4^{[8]} \\
\pm 3.1\end{array}$ & & & \\
\hline XVI & Diethylmercury, $\mathrm{C}_{4} \mathrm{H}_{10} \mathrm{Hg}, 28$ & & & $\begin{array}{l}44.8^{[5]} \\
\pm 1.7\end{array}$ & $\begin{array}{l}3103.3 \\
{[5]} \\
\pm 4.2\end{array}$ & $\begin{array}{l}-30.2 \\
{[8]} \\
\pm 1.5\end{array}$ & $\begin{array}{l}90.0^{\mathrm{c}} \\
\pm 0.5\end{array}$ & & & $\begin{array}{l}182.3 \\
{[11]}\end{array}$ \\
\hline XVII & Dipropylmercury, $\mathrm{C}_{6} \mathrm{H}_{14} \mathrm{Hg}, 40$ & & & $\begin{array}{l}55.2^{[5]} \\
\pm 1.3\end{array}$ & $\begin{array}{l}4341.3 \\
{[5] \quad \pm 4.2}\end{array}$ & $\begin{array}{l}24.9^{[8]} \\
\pm 5.0\end{array}$ & $\begin{array}{l}-30.3^{[8]} \\
\pm 5.2\end{array}$ & & & \\
\hline XVIII & $\begin{array}{l}\text { Di-(2-methylethyl)mercury, } \\
\mathrm{C}_{6} \mathrm{H}_{14} \mathrm{Hg}, 40\end{array}$ & & & $\begin{array}{l}53.6^{[5]} \\
\pm 1.7\end{array}$ & $\begin{array}{l}4399.5 \\
{[5] \quad \pm 4.2}\end{array}$ & $\begin{array}{l}16.7^{[8]} \\
\pm 6.0\end{array}$ & $\begin{array}{l}-91.2^{[8]} \\
\pm 4.6\end{array}$ & & & \\
\hline XIX & Dibuthylmercury, $\mathrm{C}_{8} \mathrm{H}_{18} \mathrm{Hg}, 52$ & & & $\begin{array}{l}65.3^{[5]} \\
\pm 4.2\end{array}$ & $\begin{array}{l}5622.9 \\
{[5]} \\
\pm 6.3\end{array}$ & $\begin{array}{l}97.7^{[8]} \\
\pm 6.4\end{array}$ & $\begin{array}{l}32.4^{[8]} \\
\pm 7.7^{-1}\end{array}$ & & & \\
\hline $\mathrm{XX}$ & $\begin{array}{l}\text { Di-(2-methylpropyl)mercury, } \\
\mathrm{C}_{8} \mathrm{H}_{18} \mathrm{Hg}, 52\end{array}$ & & & $\begin{array}{l}63.6^{[5]} \\
\pm 4.2\end{array}$ & $\begin{array}{l}5622.9 \\
{[5] \quad \pm 6.3}\end{array}$ & $\begin{array}{l}101.9 \\
{[8]} \\
\pm 6.4\end{array}$ & $\begin{array}{l}38.3^{[8]} \\
\pm 7.7^{-1}\end{array}$ & & & \\
\hline XXI & $\begin{array}{l}\text { Di-(2-methylbuthyl)mercury, } \\
\mathrm{C}_{10} \mathrm{H}_{21} \mathrm{Hg}, 64\end{array}$ & & & $\begin{array}{l}71.1^{[5]} \\
\pm 6.3\end{array}$ & $\begin{array}{l}6925.4 \\
{[5] \quad \pm 8.4}\end{array}$ & $\begin{array}{l}153.8 \\
{[8]} \\
\pm 8.5\end{array}$ & $\begin{array}{l}83.0^{[8]} \\
\pm 11.0\end{array}$ & & & \\
\hline XXII & Diphenylmercury, $\mathrm{C}_{12} \mathrm{H}_{10} \mathrm{Hg}, 60$ & & & $\begin{array}{l}112.8^{[5]} \\
\pm 0.8\end{array}$ & $\begin{array}{l}6431.2 \\
{ }_{[5]}^{5]} \pm 7.5\end{array}$ & $\begin{array}{l}300.0^{c} \\
\pm 8.0\end{array}$ & $\begin{array}{l}410.0^{\mathrm{c}} \\
\pm 8.0\end{array}$ & & & $\begin{array}{l}225.5 \\
{[12]}\end{array}$ \\
\hline \multicolumn{11}{|c|}{ Bor compounds } \\
\hline XXIII & Trimethylborane, $\mathrm{C}_{3} \mathrm{H}_{9} \mathrm{~B}, 24$ & & & $\begin{array}{l}20.2^{[5]} \\
\pm 0.1\end{array}$ & $\begin{array}{l}2989.5 \\
{[5] \pm 22.6}\end{array}$ & $\begin{array}{l}142.7 \\
{[5]} \\
\pm 22.7\end{array}$ & $\begin{array}{l}122.6^{[5]} \\
\pm 22.7\end{array}$ & & & \\
\hline XXIV & Triethylborane, $\mathrm{C}_{6} \mathrm{H}_{15} \mathrm{~B}, 42$ & $\begin{array}{l}4861.3 \\
\pm 24.3\end{array}$ & $\begin{array}{l}287.5 \\
\pm 1.4\end{array}$ & $\begin{array}{l}36.8^{[5]} \\
\pm 0.4\end{array}$ & $\begin{array}{l}4975.6 \\
{[5] \pm 15.1}\end{array}$ & $\begin{array}{l}189.1 \\
{[5]} \\
\pm 5.0\end{array}$ & $\begin{array}{l}152.3^{[5]} \\
\pm 5.0\end{array}$ & $\begin{array}{l}330.05 \\
{[13]}\end{array}$ & $\begin{array}{l}383.8 \\
\pm 1.9\end{array}$ & $\begin{array}{l}240.0 \\
{[13]}\end{array}$ \\
\hline XXV & Tripropylborane, $\mathrm{C}_{9} \mathrm{H}_{21} \mathrm{~B}, 60$ & & & $\begin{array}{l}41.8^{[5]} \\
\pm 1.2\end{array}$ & $\begin{array}{l}6901.1 \\
{ }^{[5]} \pm 13.0\end{array}$ & $\begin{array}{l}277.8 \\
{[5]} \\
\pm 13.8\end{array}$ & $\begin{array}{l}236.0^{[5]} \\
\pm 13.8\end{array}$ & & & \\
\hline XXVI & Tributhylborane, $\mathrm{C}_{12} \mathrm{H}_{27} \mathrm{~B}, 78$ & & & $\begin{array}{l}61.9^{[5]} \\
\pm 2.1\end{array}$ & $\begin{array}{l}8893.5 \\
{[5] \pm 2.1}\end{array}$ & $\begin{array}{l}352.7 \\
{[5]} \\
\pm 2.9\end{array}$ & $\begin{array}{l}290.8^{[5]} \\
\pm 3.8\end{array}$ & & & \\
\hline XXVII & $\begin{array}{l}\text { Tris-(2-methylpropyl)borane, } \\
\mathrm{C}_{12} \mathrm{H}_{27} \mathrm{~B}, 78\end{array}$ & & & $\begin{array}{l}59.8^{[5]} \\
\pm 2.1\end{array}$ & $\begin{array}{l}8833.7 \\
{ }^{[5]} \pm 5.4\end{array}$ & $\begin{array}{l}383.2 \\
{[5]} \\
\pm 6.3\end{array}$ & $\begin{array}{l}323.4^{[5]} \\
\pm 6.7\end{array}$ & & & \\
\hline XXVIII & $\begin{array}{l}\text { Tris-(3-methylpropyl)borane, } \\
\mathrm{C}_{12} \mathrm{H}_{27} \mathrm{~B}, 78\end{array}$ & & & $\begin{array}{l}60.7^{[5]} \\
\pm 2.1\end{array}$ & $\begin{array}{l}8811.9 \\
{[5] \pm 25.1}\end{array}$ & $\begin{array}{l}305.4 \\
{[5]} \\
\pm 25.1\end{array}$ & $\begin{array}{l}242.7^{[5]} \\
\pm 25.1\end{array}$ & & & \\
\hline XXIX & $\begin{array}{l}\text { Tris-(2-methylbuthyl)borane, } \\
\mathrm{C}_{15} \mathrm{H}_{33} \mathrm{~B}, 96\end{array}$ & & & $\begin{array}{l}72.0^{[5]} \\
\pm 2.5\end{array}$ & $\begin{array}{l}10801.8 \\
{[5] \pm 6.7}\end{array}$ & $\begin{array}{l}453.1 \\
{[5]} \\
\pm 7.9\end{array}$ & $\begin{array}{l}381.2^{[5]} \\
\pm 8.4\end{array}$ & & & \\
\hline \multicolumn{11}{|c|}{ Aluminum compounds } \\
\hline $\mathrm{XXX}$ & $\begin{array}{l}\text { Trimethylaluminum, } \mathrm{C}_{3} \mathrm{H}_{9} \mathrm{Al} \text {, } \\
24\end{array}$ & $\begin{array}{l}2911.6 \\
\pm 14.5\end{array}$ & $\begin{array}{l}182.6 \\
\pm 0.9\end{array}$ & $\begin{array}{l}63.2^{[14]} \\
\pm 1.7\end{array}$ & $\begin{array}{l}3184.4 \\
{[5] \quad \pm 9.6}\end{array}$ & 120.2 & $\begin{array}{l}57.0^{[8]} \\
\pm 9.7\end{array}$ & $\begin{array}{l}209.4 \\
{[14]}\end{array}$ & & $\begin{array}{l}155.6 \\
{[14]}\end{array}$ \\
\hline
\end{tabular}




\begin{tabular}{|c|c|c|c|c|c|c|c|c|c|c|}
\hline \multirow[b]{2}{*}{$\begin{array}{l}\text { No } \\
\text { comp. }\end{array}$} & \multirow[b]{2}{*}{$\begin{array}{l}\text { Title of Compound, formula, } \\
N\end{array}$} & \multicolumn{2}{|c|}{ Free energy } & \multicolumn{4}{|c|}{ Enthalpy (heat contributions) } & \multicolumn{3}{|c|}{ Entropy and heat capacity } \\
\hline & & $\begin{array}{l}-\Delta_{\mathrm{c}} G^{0} \\
(\text { Calc. } \\
\pm 0.5)^{\text {a }}\end{array}$ & $\begin{array}{l}-\Delta_{\mathrm{f}} G^{\mathrm{o}} \\
(\text { Calc. } \\
\pm 0.5)^{\mathrm{b}}\end{array}$ & $-\Delta_{\text {vap }} H^{\mathrm{o}}$ & $-\Delta_{\mathrm{c}} H^{\mathrm{o}}$ & $-\Delta_{\mathrm{f}} H^{\mathrm{o}}$ & $-\Delta_{\mathrm{f}} H_{\mathrm{gas}}^{\mathrm{o}}$ & $S^{\mathrm{o}}$ & $\begin{array}{l}-\Delta_{\triangle} S^{\mathrm{o}} \\
(\text { Calc. } \\
\pm 0.5)^{\mathrm{f}}\end{array}$ & $C_{p}$ \\
\hline 1 & 2 & 3 & 4 & 5 & 6 & $\begin{array}{l}7 \\
\pm 9.6\end{array}$ & 8 & 9 & 10 & 11 \\
\hline XXXI & Triethylaluminum, $\mathrm{C}_{6} \mathrm{H}_{15} \mathrm{Al}, 42$ & $\begin{array}{l}5088.0 \\
\pm 6.3\end{array}$ & $\begin{array}{l}309.0 \\
\pm 1.5\end{array}$ & $\begin{array}{l}73.2^{[5]} \\
\pm 2.1\end{array}$ & $\begin{array}{l}5125.4 \\
{[5] \quad \pm 8.8}\end{array}$ & $\begin{array}{l}217.2 \\
{[8]} \\
\pm 8.9\end{array}$ & $\begin{array}{l}144.0^{[8]} \\
\pm 9.1\end{array}$ & $\begin{array}{l}308.0 \\
{[15]}\end{array}$ & $\begin{array}{l}1255.1 \\
\pm 6.3\end{array}$ & $\begin{array}{l}239.0 \\
{[15]}\end{array}$ \\
\hline XXXII & $\begin{array}{l}\text { Tripropylaluminum, } \mathrm{C}_{9} \mathrm{H}_{21} \mathrm{Al} \text {, } \\
54\end{array}$ & $\begin{array}{l}7010.7 \\
\pm 35.0\end{array}$ & $\begin{array}{l}432.6 \\
\pm 2.2\end{array}$ & $\begin{array}{l}42.5^{[5]} \\
\pm 1.2\end{array}$ & $\begin{array}{l}7058.4 \\
\quad \pm 0.4\end{array}$ & $\begin{array}{l}322.3 \\
{[8]} \\
\pm 1.5\end{array}$ & $\begin{array}{l}279.8^{[8]} \\
\pm 1.9\end{array}$ & $\begin{array}{l}370.1 \\
{[16]}\end{array}$ & $\begin{array}{l}1600.1 \\
\pm 8.0\end{array}$ & $\begin{array}{l}340.5 \\
{[16]}\end{array}$ \\
\hline XXXIII & $\begin{array}{l}\text { Tributhylaluminum, } \mathrm{C}_{12} \mathrm{H}_{27} \mathrm{Al} \text {, } \\
78\end{array}$ & & & & $\begin{array}{l}9046.2 \\
{[5] \pm 5.4}\end{array}$ & $\begin{array}{l}372.5 \\
{[8]} \\
\pm 2.2\end{array}$ & & & & \\
\hline XXXIV & $\begin{array}{l}\text { Tris-(2- } \\
\text { methylpropyl)aluminum, } \\
\mathrm{C}_{12} \mathrm{H}_{27} \mathrm{Al}, 78\end{array}$ & & & $61.1^{[17]}$ & $\begin{array}{l}9030.3 \\
{[5]} \\
\pm 7.5\end{array}$ & $\begin{array}{l}388.4 \\
{[8]} \\
\pm 7.7\end{array}$ & $327.3^{[8]}$ & & & \\
\hline \multicolumn{11}{|c|}{ Germanium compounds } \\
\hline XXXV & $\begin{array}{l}\text { Tetramethylgermanium, } \\
\mathrm{C}_{4} \mathrm{H}_{12} \mathrm{Ge}, 32\end{array}$ & $\begin{array}{l}3525.1 \\
\pm 17.6\end{array}$ & $\begin{array}{l}223.3 \\
\pm 1.1\end{array}$ & $\begin{array}{l}27.3^{[18]} \\
\pm 0.4^{-1}\end{array}$ & $\begin{array}{l}3708.9 \\
{[17]} \\
\pm 6.3\end{array}$ & $\begin{array}{l}134.8 \\
{[8]} \\
\pm 6.4\end{array}$ & $\begin{array}{l}107.5^{[8]} \\
\pm 6.4\end{array}$ & $\begin{array}{l}296.8 \\
{[19]}\end{array}$ & $\begin{array}{l}616.6 \\
\pm 3.1\end{array}$ & $\begin{array}{l}196.9 \\
{[19]}\end{array}$ \\
\hline XXXVI & $\begin{array}{l}\text { Tetraethylgermanium, } \mathrm{C}_{8} \mathrm{H}_{20} \mathrm{Ge} \text {, } \\
56\end{array}$ & $\begin{array}{l}6105.0 \\
\pm 30.5\end{array}$ & $\begin{array}{l}338.4 \\
\pm 1.7\end{array}$ & $\begin{array}{l}45.7^{[21]} \\
\pm 0.4\end{array}$ & $\begin{array}{l}6356.8 \\
{[5] \quad \pm 3.2}\end{array}$ & $\begin{array}{l}210.6 \\
{[8]} \\
\pm 6.5\end{array}$ & $\begin{array}{l}156.8^{[8]} \\
\pm 4.8\end{array}$ & $\begin{array}{l}428.8 \\
{[20]}\end{array}$ & $\begin{array}{l}843.6 \\
\pm 3.1\end{array}$ & $\begin{array}{l}294.7 \\
{[20]}\end{array}$ \\
\hline $\begin{array}{l}\text { XXXVI } \\
\text { I }\end{array}$ & $\begin{array}{l}\text { Tetrapropylgermanium, } \\
\mathrm{C}_{12} \mathrm{H}_{28} \mathrm{Ge}, 80\end{array}$ & & & $\begin{array}{l}61.5^{[21]} \\
\pm 4.2\end{array}$ & $\begin{array}{l}8972.6 \\
{[5] \quad \pm 2.0}\end{array}$ & $\begin{array}{l}290.1 \\
{[8]} \\
\pm 3.3\end{array}$ & $\begin{array}{l}228.6^{[8]} \\
\pm 5.3\end{array}$ & & & \\
\hline $\begin{array}{l}\text { XXXVI } \\
\text { II }\end{array}$ & $\begin{array}{l}\text { Tetraphenylgermanium, } \\
\mathrm{C}_{24} \mathrm{H}_{20} \mathrm{Ge}, 120\end{array}$ & & & $\begin{array}{l}164.0^{\mathrm{d}} \\
\pm 4.2\end{array}$ & $\begin{array}{l}13139.0 \\
{[17]} \\
\pm 14.0\end{array}$ & $\begin{array}{l}-602.0 \\
{[8]} \\
\pm 10.0\end{array}$ & $\begin{array}{l}-438.0^{[8]} \\
\pm 15.0\end{array}$ & & & \\
\hline \multicolumn{11}{|c|}{ Stannum compounds } \\
\hline XXXIX & $\begin{array}{l}\text { Tetramethylstannane, } \mathrm{C}_{4} \mathrm{H}_{12} \mathrm{Sn} \text {, } \\
32 \\
\text { Tetraethylstannane, } \mathrm{C}_{8} \mathrm{H}_{20} \mathrm{Sn} \text {, } \\
56\end{array}$ & $\begin{array}{l}3633.0 \\
\pm 18.2\end{array}$ & $\begin{array}{l}142.7 \\
\pm 0.7\end{array}$ & $\begin{array}{l}32.0^{[18]} \\
\pm 0.8^{[18]} \\
50.6^{18]} \\
\pm 0.2\end{array}$ & $\begin{array}{l}3820.0^{\mathrm{c}} \\
\pm 90.0^{\circ} \\
6489.4 \\
{[5] \quad \pm 8.4}\end{array}$ & $\begin{array}{l}50.0^{\mathrm{e}} \\
\pm 80.0^{[8]} \\
92.6^{8]} \\
\pm 2.6\end{array}$ & $\begin{array}{l}20.0^{\mathrm{e}} \\
\pm 80.0^{[8]} \\
42.0^{[8]} \\
\pm 2.6\end{array}$ & $\begin{array}{l}310.8 \\
{[22]}\end{array}$ & $\begin{array}{l}627.0 \\
\pm 3.1\end{array}$ & $\begin{array}{l}197.9 \\
{[22]} \\
301.7 \\
{[23]}\end{array}$ \\
\hline XLI & $\begin{array}{l}\text { Tetrapropylstannane, } \mathrm{C}_{12} \mathrm{H}_{28} \mathrm{Sn} \text {, } \\
80\end{array}$ & & & $\begin{array}{l}65.4^{[18]} \\
\pm 2.5\end{array}$ & $\begin{array}{l}9093.1 \\
{[5] \quad \pm 5.0}\end{array}$ & $\begin{array}{l}208.3 \\
{[8]} \\
\pm 5.3\end{array}$ & $\begin{array}{l}142.9^{[8]} \\
\pm 5.9\end{array}$ & & & \\
\hline XLII & $\begin{array}{l}\text { Tetrakis-(1- } \\
\text { methylethyl)stannane, } \\
\mathrm{C}_{12} \mathrm{H}_{28} \mathrm{Sn}, 80\end{array}$ & & & $\begin{array}{l}64.9^{[17]} \\
\pm 4.2\end{array}$ & $\begin{array}{l}9096.4 \\
{[5] \quad \pm 3.8}\end{array}$ & $\begin{array}{l}184.5 \\
{[8]} \\
\pm 5.7\end{array}$ & $\begin{array}{l}119.6^{[8]} \\
\pm 7.1\end{array}$ & & & \\
\hline XLIII & $\begin{array}{l}\text { Tetrabuthylstannane, } \mathrm{C}_{16} \mathrm{H}_{36} \mathrm{Sn}, \\
104\end{array}$ & & & $\begin{array}{l}82.8^{\mathrm{b}} \\
\pm 2.1\end{array}$ & $\begin{array}{r}11717.3 \\
{[5] \quad \pm 6.7}\end{array}$ & $\begin{array}{l}300.2 \\
{[8]} \\
\pm 4.0\end{array}$ & $\begin{array}{l}217.4^{[8]} \\
\pm 4.5\end{array}$ & & & \\
\hline XLIV & $\begin{array}{l}\text { Tetrakis-(1- } \\
\text { methylpropyl)stannane, } \\
\mathrm{C}_{16} \mathrm{H}_{36} \mathrm{Sn}, 104\end{array}$ & & & & $\begin{array}{r}11690.9 \\
\quad \pm 5.9\end{array}$ & $\begin{array}{l}327.8 \\
{[8]} \\
\pm 6.3\end{array}$ & & & & \\
\hline XLV & $\begin{array}{l}\text { Tetraphenylstannane, } \mathrm{C}_{24} \mathrm{H}_{20} \mathrm{Sn}, \\
120\end{array}$ & & & $\begin{array}{l}161.1 \\
{[24]} \\
\pm 4.2\end{array}$ & $\begin{array}{r}13566.6 \\
{[5] \quad \pm 7.1}\end{array}$ & $\begin{array}{l}-412.3 \\
{[5]} \\
\pm 3.6\end{array}$ & $\begin{array}{l}-478.6^{[5]} \\
\pm 4.0\end{array}$ & & & $\begin{array}{l}426.3 \\
{[12]}\end{array}$ \\
\hline \multicolumn{11}{|c|}{ Lead compounds } \\
\hline XLVI & Tetramethyllead, $\mathrm{C}_{4} \mathrm{H}_{12} \mathrm{~Pb}, 32$ & $\begin{array}{l}3529.7 \\
\pm 17.6\end{array}$ & $\begin{array}{l}193.5 \\
\pm 1.0\end{array}$ & $\begin{array}{l}38.0^{[5]} \\
\pm 0.4\end{array}$ & $\begin{array}{l}3711.2 \\
{[25]} \\
\pm 1.3\end{array}$ & $\begin{array}{l}-98.1 \\
{[8]} \\
\pm 4.4\end{array}$ & $\begin{array}{l}-136.1^{[8]} \\
\pm 4.4\end{array}$ & $\begin{array}{l}320.0 \\
{[25]}\end{array}$ & $\begin{array}{l}608.6 \\
\pm 3.0\end{array}$ & $\begin{array}{l}202.5 \\
{[25]}\end{array}$ \\
\hline XLVII & Tetraethyllead, $\mathrm{C}_{8} \mathrm{H}_{20} \mathrm{~Pb}, 56$ & $\begin{array}{l}6375.2 \\
\pm 31.9\end{array}$ & $\begin{array}{l}191.5 \\
\pm 1.0\end{array}$ & $\begin{array}{l}56.6^{[18]} \\
\pm 1.0\end{array}$ & $\begin{array}{l}6383.5 \\
{[25]} \\
\pm 2.5\end{array}$ & $\begin{array}{l}-53.0 \\
{[8]} \\
\pm 5.0\end{array}$ & $\begin{array}{l}-109.6^{[8]} \\
\pm 5.1\end{array}$ & $\begin{array}{l}464.6 \\
{[15]}\end{array}$ & $\begin{array}{l}27.8 \\
\pm 0.1\end{array}$ & $\begin{array}{l}307.4 \\
{[15]}\end{array}$ \\
\hline XLVIII & Tetrapropyllead, $\mathrm{C}_{12} \mathrm{H}_{28} \mathrm{~Pb}, 80$ & & & & $\begin{array}{l}9055.8 \\
{[4] \pm 45.3}\end{array}$ & & & & & \\
\hline XLIX & Tetraphenyllead, $\mathrm{C}_{24} \mathrm{H}_{20} \mathrm{~Pb}, 120$ & & & $\begin{array}{l}159.0 \\
{[26]}\end{array}$ & $\begin{array}{l}13036.0 \\
{[17]}\end{array}$ & $\begin{array}{l}-515.0 \\
{[8]}\end{array}$ & $\begin{array}{l}-674.0^{[8]} \\
\pm 15.0\end{array}$ & & & \\
\hline & & & & \pm 1.0 & \pm 15.0 & \pm 15.0 & & & & \\
\hline
\end{tabular}

${ }^{a}$ Calc. through Eq. (11); ${ }^{\mathrm{b}}$ Calc. through Eq. (12); ${ }^{\mathrm{c}}$ Average of 6-7 values; ${ }^{\mathrm{d}}$ Calculated as a difference between $\Delta_{\mathrm{f}} \mathrm{H}^{\mathrm{o}}$ cond $-\Delta_{\mathrm{f}} \mathrm{H}^{\mathrm{o}}$ gas $;{ }^{\mathrm{e}}$ Data compiled from http://webbok.nist.gov/chemistry; ${ }^{\mathrm{f}}$ Calc. through Eq. (10). 
$\Delta \mathrm{cSocondP}=\Sigma$ ni SoproductsP-P $\Sigma$ nj Soreactants (10) here ni and nj are stoichiometric coefficients.

$$
\begin{aligned}
& \Delta \mathrm{cGo}=\Delta \mathrm{cHo}-298.15 \cdot \Delta \mathrm{cSo} \\
& \Delta \mathrm{fGo}=\Delta \mathrm{fHo}-298.15 \cdot \text { So }
\end{aligned}
$$

\subsubsection{Cadmium Compounds}

Thermodynamics with cadmium organic compounds it is similar to a situation with Zn-compounds. Data on entropy and the heat of capacity in condensed state are known; on this basis the values of free energies of combustion and formation $\left(\Delta_{\mathrm{c}, \mathrm{f}} G^{\mathrm{o}}\right)$ for compound (XI, Table 1) are calculated (Eqs. 11 and 12).

The Eqs. (13 and 14) for heats of vaporization and combustion according to for compounds (XI-XIV) are calculated also (Table 2).

$$
\begin{aligned}
& \Delta_{\text {vap }} H^{\mathrm{o}}=(22.9 \pm 2.8)+(0.8 \pm 0.07) N r 0.993, S_{\mathrm{o}} 1.9, n 4 \text { (compounds XI-XIV) } \\
& \Delta_{\mathrm{c}} H^{\mathrm{o}}=(174.4 \pm 3.6)-(113.4 \pm 0.02) N r 0.999, S_{\mathrm{o}} 26.7, n 3 \text { (compounds XI-XIII) }
\end{aligned}
$$

\begin{tabular}{|c|c|c|c|c|c|c|}
\hline $\begin{array}{l}\text { The functions } \\
\text { of processes }\end{array}$ & $\begin{array}{l}\text { No Eq. in } \\
\text { text }\end{array}$ & $i$ & $f$ & $r$ & $S_{o}$ & $\begin{array}{l}\text { Nos. of compounds in Table } \\
1\end{array}$ \\
\hline 1 & 2 & 3 & 4 & 5 & 6 & 7 \\
\hline \multicolumn{7}{|c|}{ Lithium compounds } \\
\hline$\Delta_{\mathrm{c}} H^{\mathrm{o}}$ & 4 & $-249.6 \pm 28.8$ & $-106.9 \pm 1.4$ & 0.999 & 11.9 & I-III \\
\hline$\Delta_{\mathrm{f}} H_{\text {cond }}^{\mathrm{o}}$ & 5 & $-1053.1 \pm 87.9$ & $36.8 \pm 3.2$ & 0.999 & 11.3 & III-VI \\
\hline \multicolumn{7}{|l|}{ Zinc compounds } \\
\hline$\Delta_{\mathrm{vap}} H^{\mathrm{o}}$ & 6 & $19.7 \pm 2.1$ & $0.7 \pm 0.05$ & 0.993 & 1.5 & VII-X \\
\hline$\Delta_{\mathrm{c}} H^{\mathrm{o}}$ & 7 & $-286.5 \pm 29.5$ & $-109.3 \pm 0.8$ & 0.999 & 21.6 & VII-X \\
\hline$\Delta_{\mathrm{f}} H_{\text {cond }}^{\mathrm{o}}$ & 8 & $102.7 \pm 28.9$ & $-3.9 \pm 0.8$ & 0.961 & 21.2 & VII-X \\
\hline$\Delta_{\mathrm{f}} H_{\text {gas }}^{\mathrm{o}}$ & 9 & $122.1 \pm 28.9$ & $-3.3 \pm 0.9$ & 0.937 & 23.4 & VII-X \\
\hline \multicolumn{7}{|c|}{ Cadmium compounds } \\
\hline$\Delta_{\mathrm{vap}} H^{\mathrm{o}}$ & 13 & $22.9 \pm 2.8$ & $0.8 \pm 0.07$ & 0.993 & 1.9 & XI-XIV \\
\hline$\Delta_{\mathrm{c}} H^{\mathrm{o}}$ & 14 & $174.4 \pm 3.6$ & $-113.4 \pm 0.02$ & 0.999 & 26.7 & XI-XIII \\
\hline \multicolumn{7}{|c|}{ Mercury compounds } \\
\hline$\Delta_{\mathrm{vap}} H^{\mathrm{o}}$ & 15 & $22.9 \pm 1.4$ & $0.8 \pm 0.03$ & 0.995 & 1.3 & XV-XXI \\
\hline$\Delta_{\mathrm{c}} H^{\mathrm{o}}$ & 16 & $-131.1 \pm 31.2$ & $-105.7 \pm 0.7$ & 0.999 & 28.8 & XV-XXII \\
\hline$\Delta_{\mathrm{f}} H_{\text {cond }}^{\mathrm{o}}$ & 17 & $148.8 \pm 17.6$ & $-4.7 \pm 0.4$ & 0.986 & 15.4 & XV, XVI, XVIII-XXI \\
\hline$\Delta_{\mathrm{f}} H_{\text {gas }}^{\mathrm{o}}$ & 18 & $191.7 \pm 43.7$ & $-4.1 \pm 1.0$ & 0.903 & 38.4 & XV, XVI, XVIII-XXI \\
\hline \multicolumn{7}{|l|}{ Bor compounds } \\
\hline$\Delta_{\mathrm{vap}} H^{\circ}$ & 21 & $3.5 \pm 3.2$ & $0.7 \pm 0.05$ & 0.990 & 2.8 & XXIII-XXIX \\
\hline$\Delta_{\mathrm{c}} H^{\mathrm{o}}$ & 22 & $-405.4 \pm 33.6$ & $-108.3 \pm 0.5$ & 0.999 & 29.6 & XXIII-XXIX \\
\hline$\Delta_{\mathrm{f}} H_{\text {cond }}^{\mathrm{o}}$ & 23 & $-24.5 \pm 32.9$ & $-4.2 \pm 0.5$ & 0.970 & 28.9 & XXIII-XXIX \\
\hline$\Delta_{\mathrm{f}} H_{\text {gas }}^{\mathrm{o}}$ & 24 & $-21.2 \pm 34.7$ & $-3.5 \pm 0.5$ & 0.952 & 30.5 & XXIII-XXIX \\
\hline \multicolumn{7}{|c|}{ Aluminum compounds } \\
\hline$\Delta_{\mathrm{c}} G^{\mathrm{o}}$ & 25 & $412.4 \pm 450.1$ & $-135.4 \pm 10.7$ & 0.997 & 229.6 & XXX-XXXII \\
\hline$\Delta_{\mathrm{f}} G_{\text {cond }}^{\mathrm{o}}$ & 26 & $21.1 \pm 37.5$ & $-8.2 \pm 0.9$ & 0.994 & 19.1 & XXX-XXXII \\
\hline$\Delta_{\mathrm{c}} H^{\mathrm{o}}$ & 27 & $-720.4 \pm 406.9$ & $-108.1 \pm 6.9$ & 0.994 & 322.5 & XXX-XXXIV \\
\hline$\Delta_{\mathrm{f}} H_{\text {cond }}^{\mathrm{o}}$ & 28 & $-23.2 \pm 37.2$ & $-4.7 \pm 0.6$ & 0.974 & 29.4 & XXX-XXXIV \\
\hline$\Delta_{\mathrm{f}} H_{\text {gas }}^{\mathrm{o}}$ & 29 & $128.6 \pm 74.2$ & $-7.2 \pm 1.8$ & 0.971 & 37.9 & XXX-XXXII \\
\hline$S_{\text {cond }}^{o}$ & 30 & $81.2 \pm 3.5$ & $5.4 \pm 0.1$ & 0.999 & 1.8 & XXX-XXXII \\
\hline$\Delta_{\mathrm{c}} S_{\text {cond }}^{\mathrm{o}}$ & 31 & $-355.7 \pm 112.8$ & $-22.5 \pm 2.7$ & 0.993 & 57.5 & XXX-XXXII \\
\hline \multicolumn{7}{|c|}{ Germanium compounds } \\
\hline$\Delta_{\mathrm{vap}} H^{\mathrm{o}}$ & 33 & $-32.8 \pm 25.8$ & $1.5 \pm 0.3$ & 0.953 & 21.3 & XXXV-XXXVIII \\
\hline$\Delta_{\mathrm{c}} H^{\mathrm{o}}$ & 34 & $-333.2 \pm 88.3$ & $-107.1 \pm 1.1$ & 0.999 & 72.2 & XXXV-XXXVIII \\
\hline$\Delta_{\mathrm{f}} H_{\text {cond }}^{\mathrm{o}}$ & 35 & $-30.6 \pm 2.6$ & $-3.2 \pm 0.05$ & 0.999 & 1.5 & XXXV-XXXVII \\
\hline \multicolumn{7}{|c|}{ Stannum compounds } \\
\hline$\Delta_{\mathrm{vap}} H^{\mathrm{o}}$ & 36 & $10.3 \pm 1.4$ & $0.7 \pm 0.02$ & 0.998 & 1.1 & XXXIX-XLIII \\
\hline$\Delta_{\mathrm{c}} H^{\mathrm{o}}$ & 37 & $-299.0 \pm 88.3$ & $-110.0 \pm 0.5$ & 0.999 & 40.6 & XXXIX-XLV \\
\hline$\Delta_{\mathrm{f}} H_{\text {cond }}^{\mathrm{o}}$ & 38 & $97.7 \pm 32.2$ & $-3.8 \pm 0.4$ & 0.979 & 25.2 & XXXIX-XLIV \\
\hline$\Delta_{\mathrm{f}} H_{\text {gas }}^{\mathrm{o}}$ & 39 & $90.3 \pm 31.2$ & $-2.8 \pm 0.4$ & 0.969 & 22.9 & XXXIX-XLIII \\
\hline \multicolumn{7}{|c|}{ Lead compounds } \\
\hline$\Delta_{\mathrm{vap}} H^{\circ}$ & 40 & $-14.0 \pm 13.9$ & $1.4 \pm 0.2$ & 0.992 & 11.4 & XLVI, XLVII, XLIX \\
\hline$\Delta_{\mathrm{c}} H^{\mathrm{o}}$ & 41 & $-417.2 \pm 184.0$ & $-106.0 \pm 2.3$ & 0.999 & 151.4 & XLVI-XLIX \\
\hline$\Delta_{\mathrm{f}} H_{\text {cond }}^{\mathrm{o}}$ & 42 & $-141.9 \pm 153.3$ & $5.2 \pm 1.9$ & 0.937 & 125.4 & XLVI, XLVII,X LIX \\
\hline$\Delta_{\mathrm{f}} H_{\text {gas }}^{\mathrm{o}}$ & 43 & $-155.9 \pm 167.2$ & $6.7 \pm 2.1$ & 0.953 & 136.8 & XLVI, XLVII, XLIX \\
\hline
\end{tabular}

Table 2. The parameters of equations $\Delta \Psi^{(0)}\left(\mathrm{kJ} \mathrm{mol}^{-1}\right.$ and $\left.J \mathrm{~mol}^{-1} \mathrm{~K}^{-1}\right)=i \pm f * N$ for vaporization, combustion, formation of organometallic compounds; $P$ $101 \mathrm{kPa}$; T $298.15 \mathrm{~K}$ 


\subsubsection{Mercury Compounds}

The heat contributions (enthalpies) are known for 8 compounds (XV-XXII, Table 1). These data have allowed to calculate four equations (15-18) for vaporization, combustion and formation in condensed and gaseous phases

$$
\begin{gathered}
\Delta_{\text {vap }} H^{\mathrm{o}}=(22.9 \pm 1.4)+(0.8 \pm 0.03) N r 0.995, S_{\mathrm{o}} 1.3, n 7 \text { (compounds XV-XXI) } \\
\Delta_{\mathrm{c}} H^{\mathrm{o}}=(-131.1 \pm 31.2)-(105.3 \pm 0.8) N r 0.999, S_{\mathrm{o}} 28.8, n 8 \text { (compounds XV-XXII) } \\
\Delta_{\mathrm{f}} H_{\text {cond }}^{\mathrm{o}}=(148.8 \pm 17.6)-(4.7 \pm 0.4) N r 0.986, S_{\mathrm{o}} 15.4, n 6 \text { (compounds XV, XVI, XVIII-XXI) } \\
\Delta_{\mathrm{f}} H_{\text {gas }}^{\mathrm{o}}=(191.7 \pm 43.8)-(4.1 \pm 1.0) N r 0.903, S_{\mathrm{o}} 38.4, n 6 \text { (compounds XV, XVI, XVIII-XXI) }
\end{gathered}
$$

\subsection{Organic Compounds of III Group (Bor, Aluminum)}

The processes of combustion of these types of organometallic compounds in condensed phase of III group (XXIII-XXXIV, Table 1) may be presented by the different equations. So, the combustion of organoboranes (XXIII-
XXIX) conducts to formation of boric acid, carbon dioxide and water (Eq. 19), but at the combustion of organoaluminums (XXX-XXXIV) are formed aluminum oxide, carbon dioxide and water (Eq. 20) [5, 28]

$$
\begin{aligned}
& \mathrm{C}_{\mathrm{a}} \mathrm{H}_{\mathrm{b}} \mathrm{B} \text { (cond) }+(4 \mathrm{a}+\mathrm{b}) / 2 \mathrm{O}_{2} \rightarrow \mathrm{H}_{3} \mathrm{BO}_{3} \text { (sol) }+\mathrm{a} \mathrm{CO}_{2} \text { (gas) }+\mathrm{b} / 2 \mathrm{H}_{2} \mathrm{O} \text { (liq) } \\
& 2 \mathrm{C}_{\mathrm{a}} \mathrm{H}_{\mathrm{b}} \mathrm{Al} \text { (cond) }+(4 \mathrm{a}+\mathrm{b}) / 2 \mathrm{O}_{2} \rightarrow \mathrm{Al}_{2} \mathrm{O}_{3} \text { (sol) }+2 \mathrm{CO}_{2} \text { (gas) }+\mathrm{b} \mathrm{H}_{2} \mathrm{O} \text { (liq) }
\end{aligned}
$$

to calculate four equations (21-24) for vaporization,

The thermochemical parameters are known for 7 of boranes (XXIII-XXIX, Table 1). These data have allowed combustion and formation in condensed and gaseous phases

$$
\begin{gathered}
\Delta_{\text {vap }} H^{\mathrm{o}}=(3.5 \pm 3.2)+(0.7 \pm 0.05) N r 0.990, S_{\mathrm{o}} 2.8, n 7 \text { (compounds XXIII-XXIX) } \\
\Delta_{\mathrm{c}} H^{\mathrm{o}}=(-405.4 \pm 33.6)-(108.3 \pm 0.5) N r 0.999, S_{\mathrm{o}} 29.6, n 7 \text { (compounds XXIII-XXIX) } \\
\Delta_{\mathrm{f}} H_{\text {cond }}^{\mathrm{o}}=(-24.5 \pm 32.9)-(4.2 \pm 0.5) N r 0.970, S_{\mathrm{o}} 28.9, n 7 \text { (compounds XXIII-XXIX) } \\
\Delta_{\mathrm{f}} H_{\text {gas }}^{\mathrm{o}}=(-21.2 \pm 34.7)-(3.5 \pm 0.5) N r 0.952, S_{\mathrm{o}} 30.5, n 7 \text { (compounds XXIII-XXIX) }
\end{gathered}
$$

The organic compounds of aluminum (XXX-XXXIV) have appeared more "richer" on thermocdynamic data than the previous types of compounds. The heats of vaporization, combustion, formation in condensed and gaseous phases, entropy of formation and heat capacity there are for its (Table 1). Such circumstance has allowed to calculate for compounds (XXX-XXXII) the entropies of combustion, free energies of combustion and formation (Table 1) via Eqs. (10-12) and receive seven Eqs. (25-31), reflecting the interrelations between the mentioned above thermodynamic functions and a number of valence electrons $N$ in their molecules

$$
\begin{gathered}
\Delta_{\mathrm{c}} G^{\mathrm{o}}=(412.4 \pm 450.1)-(135.4 \pm 10.7) N r 0.997, S_{\mathrm{o}} 229.6, n 3 \text { (compounds XXX-XXXII) } \\
\Delta_{\mathrm{f}} G_{\text {cond }}^{\mathrm{o}}=(21.1 \pm 37.5)-(8.2 \pm 0.9) N r 0.994, S_{\mathrm{o}} 19.1, n 3 \text { (compounds XXX-XXXII) } \\
\Delta_{\mathrm{c}} H^{\mathrm{o}}=(-720.4 \pm 406.9)-(108.1 \pm 6.9) N r 0.994, S_{\mathrm{o}} 322.5, n 5 \text { (compounds XXX-XXXIV) } \\
\Delta_{\mathrm{f}} H_{\text {cond }}^{\mathrm{o}}=(-23.2 \pm 37.2)-(4.7 \pm 0.6) N r 0.974, S_{\mathrm{o}} 29.6, n 5 \text { (compounds XXX-XXXIV) } \\
\Delta_{\mathrm{f}} H_{\text {gas }}^{\mathrm{o}}=(128.6 \pm 74.2)-(7.2 \pm 1.8) N r 0.971, S_{\mathrm{o}} 37.9, n 3 \text { (compounds XXX-XXXII) } \\
S_{\text {cond }}^{\mathrm{o}}=(81.2 \pm 3.5)+(5.4 \pm 0.1) N r 0.999, S_{\mathrm{o}} 1.8, n 3 \text { (compounds XXX-XXXII) } \\
\Delta_{\mathrm{c}} S_{\text {cond }}^{\mathrm{o}}=(-355.7 \pm 112.8)-(22.5 \pm 2.7) N r 0.993, S_{\mathrm{o}} 57.5, n 3 \text { (compounds XXX-XXXII) }
\end{gathered}
$$

\subsection{Organic Compounds of IV Group (Germanium, Stannum, Lead)}

It is necessary to note, that in the given work are not considered carbon organic compounds because of this huge class of compounds the separate paper is devoted [29]. The process of combustion of fourth group of organometallic compounds in condensed phase (XXXV-XLIX, Table 1) may be presented by the general equation (32). 


$$
\mathrm{C}_{\mathrm{a}} \mathrm{H}_{\mathrm{b}} \mathrm{Me} \text { (cond) }+(4 \mathrm{a}+\mathrm{b}) / 2 \mathrm{O}_{2} \rightarrow \mathrm{a} \mathrm{CO} \text { (gas) }+\mathrm{b} / 2 \mathrm{H}_{2} \mathrm{O}+\mathrm{MeO}_{2} \text { (sol) }
$$

here $\mathrm{Me}$ are $\mathrm{Ge}, \mathrm{Sn}, \mathrm{Pb} ; \mathrm{a}$ and $\mathrm{b}$ are stoichiometric coefficients.

\subsubsection{Germanium Compounds}

Entropy of formation $\left(S_{\text {cond }}^{o}\right)$ in condensed state is available for Ge-compounds (XXXV and XXXVI), that creates an opportunity of calculation of combustion entropy $\left(\Delta_{c} S_{\text {cond }}^{0}\right)$ and free energies of combustion and formation $\left(\Delta_{\mathrm{c}, \mathrm{f}} G^{\circ}\right)$ (Table 1) for them on the equations (10-12). The known the heats of vaporization, combustion and formation enable to receive Eqs. (33-35)

$$
\begin{aligned}
& \Delta_{\text {vap }} H^{\mathrm{o}}=(-32.8 \pm 25.8)+(1.5 \pm 0.3) N r 0.953, S_{\mathrm{o}} 21.3, n 4 \text { (compounds XXXV-XXXVIII) } \\
& \Delta_{\mathrm{c}} H^{\mathrm{o}}=(-333.2 \pm 88.3)-(107.1 \pm 1.1) N r 0.999, S_{\mathrm{o}} 72.2, n 4 \text { (compounds XXXV-XXXVIII) } \\
& \Delta_{\mathrm{f}} H_{\text {cond }}^{\mathrm{o}}=(-30.6 \pm 2.6)-(3.2 \pm 0.05) N r 0.999, S_{\mathrm{o}} 1.5, n 3 \text { (compounds XXXV-XXXVII) }
\end{aligned}
$$

\subsubsection{Stannum and Lead Compounds}

The the similar situation has turned out for organic compounds of stannum (XXXIX-XLV) and lead (XLVIXLIX). For both classes of compounds the equations of vaporization, combustion and formation in condensed and gaseous phases processes are calculated: Eqs. (36-39) for Sn-compounds and Eqs. (40-43) for $\mathrm{Pb}$-compounds

$$
\begin{gathered}
\Delta_{\text {vap }} H^{\mathrm{o}}=(10.3 \pm 1.4)+(0.7 \pm 0.02) N r 0.998, S_{\mathrm{o}} 1.1, n 5 \text { (compounds XXXIX-XLIII) } \\
\Delta_{\mathrm{c}} H^{\mathrm{o}}=(-299.0 \pm 88.3)-(110.0 \pm 0.5) N r 0.999, S_{\mathrm{o}} 40.6, n 7 \text { (compounds XXXIX-XLV) } \\
\Delta_{\mathrm{f}} H_{\text {cond }}^{\mathrm{o}}=(97.7 \pm 32.2)-(3.8 \pm 0.4) N r 0.979, S_{\mathrm{o}} 25.2, n 5 \text { (compounds XXXIX-XLIV) } \\
\Delta_{\mathrm{f}} H_{\text {gas }}^{\mathrm{o}}=(90.3 \pm 31.2)-(2.8 \pm 0.4) N r 0.969, S_{\mathrm{o}} 22.9, n 5 \text { (compounds XXXIX-XLIII) } \\
\Delta_{\text {vap }} H^{\mathrm{o}}=(-14.3 \pm 13.9)+(1.4 \pm 0.2) N r 0.992, S_{\mathrm{o}} 11.4, n 3 \text { (compounds XLVI, XLVII, XLIX) } \\
\Delta_{\mathrm{c}} H^{\mathrm{o}}=(-417.2 \pm 184.0)-(106.0 \pm 2.3) N r 0.999, S_{\mathrm{o}} 151.4, n 4 \text { (compounds XLVI-XLIX) } \\
\Delta_{\mathrm{f}} H_{\text {cond }}^{\mathrm{o}}=(-141.9 \pm 153.3)+(5.2 \pm 1.9) N r 0.937, S_{\mathrm{o}} 125.4, n 3 \text { (compounds XLVI, XLVII, XLIX) } \\
\Delta_{\mathrm{f}} H_{\text {gas }}^{\mathrm{o}}=(-155.9 \pm 164.2)+(6.7 \pm 2.1) N r 0.953, S_{\mathrm{o}} 136.8, n 3 \text { (compounds XLVI, XLVII, XLIX) }
\end{gathered}
$$

Entropy of formation $\left(S_{\text {cond }}^{\circ}\right)$ in condensed state is available for Sn-compound (XXXIX) and for $\mathrm{Pb}$ compounds (XLVI and XLVII), that creates an opportunity of calculation of combustion entropy $\left(\Delta_{c} S_{\text {cond }}^{0}\right)$ and free energies of combustion and formation $\left(\Delta_{\mathrm{c}, \mathrm{G}} G^{\mathrm{o}}\right)$ (Table 1) for them on the equations (10-12) as it has been made for organometallic compounds I-III groups.

\section{Conclusion}

It is necessary to note, that the heat of vaporization, all thermodynamic functions $\Delta_{\mathrm{c}, \mathrm{f}} G^{\mathrm{o}}, \Delta_{\mathrm{c}, \mathrm{f}} H^{\mathrm{o}}, \Delta_{\mathrm{c}} S^{\mathrm{o}}$ cond and heat capacity $\left(C_{\mathrm{p}}\right)$ organometallic compounds of I-IV groups of Mendeleev's Periodic Table can be well characterized with the number of valence electrons $N$ of them.

It is difficult to do what or the conclusions rather stoichiometric coefficients $i$ and $f$ in modified by us Kharasch equation $\Delta_{\text {vap, c, f,s }} \Psi^{0}=i \pm f^{*} N$ as they concern to organometallic compounds of the different groups. Such conclusion can be made on settlement data of I and II groups only in our opinion.

It is necessary to note, that coefficients $i$ and $f$ in the equations $(6,13,15)$ on processes vaporization of zinc, cadmium and mercury compounds are close enough among themselves (19.7 - 22.9 and $0.7-0.8$ correspondingly). The coefficientes $i$ in equations $(4,7,14,16)$, describing combustion processes differ among themselves (-131.1 174.4) a little, while f coefficients $(-105.7--109.3)$ are similar to known value $-109.0 \mathrm{~kJ} \mathrm{~mol}^{-1}$ for alkanes [3]. The coefficient $i$ essentially differs $\left(-1053.1 \mathrm{~kJ} \mathrm{~mol}^{-1}\right)$ for formation in condensed phase only for $\mathrm{Li}$-organic compounds. The same coefficients for $\mathrm{Zn}, \mathrm{Cd}$ and $\mathrm{Hg}$ compounds are close among themselves for formation processes as condensed and gaseous phases.

Probably, that such data will help with forecasting of thermochemical functions and parameters of yet not investigated organometallic compounds.

\section{References}

[1] Ovchinnikov V.V., Thermochemistry of Heteroatomic Compounds: Interdependence between of some thermochemical Parameters of the different Classes of organic nitro compounds and a Number of valence Electrons in their Molecules, ACSJ, 3, 11-23 (2013).

[2] Ovchinnikov V.V., Thermochemistry of Heteroatomic Compound: Some thermodynamic Aspects of Combustion and Formation of Carbohydrates of Different Structures, 
Chem. Journal. 2, 2, 59-65 (2013).

[3] Kharasch M.S. and Sher B., The electronic conception of valence and heats of combustion of organic compounds, J. Phys. Chem., 25, 625-658(1925).

[4] Ovchinnikov V.V., Thermochemistry of Heteroatomic Compounds: Enthalpy of Combustion of Organic Compounds of Group I-VII Elements, Doklady Physical Chemistry. 443, 49-52 (2012).

[5] CoJ.D. and Pilcher G. Thermochemistry of Organic and Organometallic Compounds. Academic Press - London and New York, pp. 640 (1970).

[6] Lebedev Yu.A. and Miroschnichenko E.A. Thermochemistry of vaporization of organic substances, Science, Moscow, pp. 215 (1981).

[7] Liebman J.F., Martino Simoes J.A. and Slaydon S.W., In Lithium Chemistry: A Theoretical And Experimental Overview, Wiley, New York: Sapse A. M. and Schleyer P. von Rague, Eds. (1995).

[8] Martinho Simões J.A., Private communication (see: http :// webbook. nist. gov/chemistry/om/).

[9] Sheiman M.S., Nistratov V.P., Kamelova G.P. and Rabinovich I.B., Low-temperature heat capacity of organic compounds of aluminum and zinc, Probl. Kalorim. Khim. Termodin., Dokl. Vses. Konf., 10th, 2, 457-459 (1984).

[10] Rabinovich I.B., Nistratov V.P., Fedoseev V.B., Sheiman M.S., Kamelova G.P., Karataev E.N. and Feshchenko I.A., Low-temperature specific heat and thermodynamic functions of diethylzinc, Zhur.Fiz. Khim. (Russ.), 62, 13491352 (1988).

[11] Burchalova G.V., Kamelova G.P., Nistratov V.P., Sheiman M.S. and Rabinovich I.B., Low-temperature heat capacity of diethylmercury, Termodin. Org. Soedin.(Russ.), 7, 21-22 (1978).

[12] Smith R.H. and Andrews D.H., Thermal energy studies. II. Phenyl derivatives of metals, J. Am. Chem. Soc., 53, 36613667 (1931).

[13] Kostryukov V.N., Samorukov O.P., Samorukova N.Kh., Krasavin A.M. and Petrunin A.B., Heat capacity of triethylboron at low temperature, Vses. Konf. Kalorim., Russia, Tezisy Dokl. 7th, 2, 387-390 (1977).

[14] McCullough J.P., Messerly J.F., Moore R.T. and Todd S.S., Trimethylaluminum: thermodynamic functions in the solid and liquid states, $0-380^{\circ} \mathrm{K}$, vapor pressure, heat of vaporization, and entropy in the ideal gas state, J. Phys. Chem., 67, 677-679 (1963).

[15] Rabinovich I.B., Nistratov V.P., Sheiman M.S., Klimov K.N., Kamelov G.P. and Zorin A.D., Specific heat and thermodynamic functions of triethylaluminium, Zhur. Fiz. Khim. (Russ.), 63, 522-x525 (1989).
[16] Sheiman M.S., Rabinovich I.B., Nistratov V.P., Smirnova N.N., Kamelova G.P., Klimov K.N. and Zorin A.D., Low temperature heat capacity and thermodynamic functions of tripropylaluminum and diisobutylaluminum, Zhur. Fiz. Khim. (Russ.), 65, 831-836 (1991).

[17] Pedley J.B. and Rylance J., Computer Analysed Thermochemical Data: Organicxand Organometallic Compounds, University of Sussex, Brigton (1977).

[18] Stull D.R., Vapor Pressure of Pure Substances Organic Compounds, Ind. Eng. Chem., 39, 517-540 (1947).

[19] Valerga A.J. and Kilpatrick J.E., Entropy related thermodynamic properties of tetramethylgermane, J. Chem. Phys., 52, 4545-4549 (1970).

[20] Rabinovich I.B., Sheiman M.S., Nistratov V.P., Kamelova G.P. and Zorin A.D., The heat capacity and thermodynamic characteristics of tetraethylgermane, Zhur. Fiz. Khim. (Russ.), 59, 2414-2417 (1985).

[21] Mogul P.H., Hochberg M.C., Michiel R., Nestel G.K., Wamsley B.L. and Coren S.D., Physical Properties of Tetran-alkylgermanes (C1-C6), J. Chem. Eng. Data, 19, 4-6 (1974).

[22] Sheiman M.S., Rabinovich I.B., Nistratov V.P., Kamelova G.P., Karataev E.N. and Feshchenko I.A., Specific heat and thermodynamic haracteristics of tetramethylstanane, Zhur. Fiz. Khim., 63, 836-838 (1989).

[23] Maslova V.A., Rabinovich I.B., Nistoalov V.P. and Faminskaya L.A., Specific heat and phase transitions of some alkyl compounds of silicon, germanium and tin, Tr. Khim. Khim. Tekhnol., (2), 44-50 (1972).

[24] Adams G.P. and Carson A.S., The Enthalpy of Combustion of Organimetallic Compounds Measured with a Vacuumjacketed Aneroud Calorimeter, The Enthalpy of Formation of Tin Tetraphenyl, J. Chem. Thermodyn., 1, 393-396 (1969).

[25] Good W.D., Scott D.W., Lacina J.L. and McCullough J.P., Tetramethyllead: heat of formation by rotating-bomb calorimetry, J. Phys. Chem., 63, 1139-1142 (1959).

[26] Stephenson R.M. and Malanowski S., Handbook of the Thermodynamics of Organic Compounds, Elsevier: New York (1987).

[27] Data for General, Organic and Physical Chemistry. Copyright by C.D. Schaeffer, Jr. (Elizabethtown College), C.A. Strausser, M.W. and Thomsen C.H. Yoder (Franklin \& Marshall College) (1989).

[28] Karapetyanz M.Kh. and Karapetyanz M.L., The basic thermodynamic constants of inorga-nic and organic chemistry, Khimia, Moskow, pp. 471 (1968).

[29] Ovchinnikov V.V., Thermochemistry of Heteroatomic Compounds: Analysis and Calculation of Some Thermodynamic Functions of Saturated Alkanes, ACSJ, in press (2013). 